\title{
ANALYSIS OF NUMERICAL SOLUTIONS OF TRANSIENT HEAT-FLOW PROBLEMS*
}

\author{
BY \\ CLARENCE M. FOWLER \\ U. S. Naval Academy
}

1. Introduction. The purpose of this paper is to present formal methods for establishing the convergence of numerical solutions of transient heat-flow problems, and to derive expressions for these solutions in terms of the initial temperatures and boundary values.

In general, heat-flow problems are classified under two groups, steady-state flow and transient flow. Steady-state problems are solved numerically by the relaxation method. Many papers dealing with the actual numerical work have been written, and Temple ${ }^{1}$ has established the validity of the relaxation method under various boundary conditions. Moskovitz ${ }^{2}$ has derived an expression in terms of the boundary temperatures for the steady-state numerical solution of a rectangular bar.

Although considerable work has been done on the actual application of numerical methods to transient heat-flow problems, very little has been written about the problems of convergence and the expression of solutions in terms of initial and boundary values. ${ }^{3}$ These last two considerations are the objects of this paper.

Two restrictions which simplify the analysis are placed on the examples considered here. First, only the one-dimensional slab is considered; secondly, the initial temperature distribution is assumed to be constant over the slab. However, by extensions of the methods used, solutions of problems concerning two- and three-dimensional rectangular objects with arbitrary initial temperature distributions are readily derived.

The various boundary conditions which have been studied include the following: the temperature at the boundary is given, and is either a constant or a function of time; the boundary is insulated; there is a constant energy input at the boundary; there is convection at the boundary. The author has made no attempt to consider all possible combinations of boundary conditions, but has tried to include enough representative cases to illustrate the methods.

The procedure followed throughout the paper has been to consider each example as a whole, and to derive solutions of the problem and take up a study of the convergence, before proceeding to the next example. In some cases solutions have been expressed in terms of a set of polynomials which are associated fundamentally with the difference equation; in other cases they have been expressed in finite Fourier series.

* Received April 11, 1945.

${ }^{1} \mathrm{G}$. Temple, The general theory of relaxation methods applied to linear systems, Proc. Roy. Soc. London (A), 169, 476-500 (1939).

2 D. Moskovitz, The numerical solutions of Laplace's and Poisson's equations, Quart. Appl. Math. 2, 148-163 (1944).

${ }^{3}$ R. Courant, K. Friedrichs and H. Lewy, Über die partiellen Differenzgleichungen der mathematischen Physik, Math. Ann. 100, 22-74 (1928). 
Properties of the polynomials needed throughout the paper have been demonstrated in an appendix.

2. The difference equation and boundary expressions. The basic one-dimensional difference equation satisfied by the numerical solutions is

where

$$
T_{x, t}=\frac{T_{x-1, t-1}+a T_{x, t-1}+T_{x+1, t-1}}{a+2},
$$

$$
\Delta t=\frac{(\Delta x)^{2}}{(a+2) \alpha} .
$$

Here, $\Delta t$ is the time interval between successive time values, $\Delta x$ is the distance between successive points across the slab, $t$ is the time (in units of $\Delta t$ ), $x$ is a space coordinate running across the slab (in units of $\Delta x), T_{x, t}$ is the temperature in the slab at time $t$ and position $x, \alpha$ is the thermal diffusivity of the material, and $a$ is the modulus of the equation.

As usually encountered, the difference equation has $a=0$ (Schmidt's equation). Dusinberre ${ }^{4}$ generalized Schmidt's equation by introducing the modulus. The value of using an arbitrary modulus lies in being able to select an arbitrary time increment as well as space increment. This is not possible in Schmidt's equation, since fixing $\Delta x$ determines $\Delta t$.

In dealing with convection, insulation, etc., at a boundary, it is always necessary to make some assumption to determine the numerical boundary expression. It should be emphasized that, for this reason there are several different expressions in use approximately representing the same boundary condition. However, it is possible to consider only one of them here, which is deduced as follows. Figure 1 shows the Fig. 1. boundary $(x=0)$ and the first two interior points of the slab. The boundary expression is derived by making a heat balance over the shaded half-segment. The heat gain by conduction throughout the time increment $\Delta t$ referred to the initial time instant $t-1$ is

$$
-h A \Delta t\left(T_{0, t-1}-T_{a}\right)+k A \Delta t \frac{\left(T_{1, t-1}-T_{0, t-1}\right)}{\Delta x},
$$

where $h$ is the surface heat transfer coefficient, $k$ is the thermal conductivity, $T_{a}$ is the ambient temperature, and $A$ is a unit area perpendicular to the slab cross section. This quantity is equated to the heat capacity gain $\frac{1}{2}(\Delta x) c \rho A\left(T_{0, t}-T_{0, t-1}\right)$, where $c$ and $\rho$ are the specific heat and density of the material, respectively. For rapid surface cooling, it is necessary to keep $\Delta x$ small, since it has been assumed above that $T_{0,8}$ will represent the temperature of the shaded segment.

After equating the two heat quantities above, and simplifying, we obtain the boundary condition

$$
T_{0, t}=\frac{2 T_{1, t-1}+(a-2 N) T_{0, t-1}+2 N T_{0}}{a+2},
$$

4 G. M. Dusinberre, Numerical methods for transient heat flow, Trans. A.S.M.E. 67, 703-709 (1945). 
where $N$, the equivalent numerical transfer coefficient, is given by the relation

$$
N=h \Delta x / k \text {. }
$$

In the case where a boundary has a constant energy input per unit area, $q$, an analysis similar to that given above yields

where

$$
T_{0, t}=\frac{2 T_{1, t-1}+a T_{0, t-1}+2 Q}{a+2},
$$

$$
Q=q \Delta x / k
$$

For an insulated boundary, $N=0$ and (2.3) becomes

$$
T_{0, t}=\frac{2 T_{1, t-1}+a T_{0, t-1}}{a+2} .
$$

For simple boundary conditions such as temperature at $x=0$ held at $u_{0}$, or temperature at $x=l$ fixed as a function of time $f(t)$, the boundary conditions are simply $T_{0, t}=u_{0}$ or $T_{l, t}=f(t)$.

3. Convergence. There are two distinct types of convergence to be considered here. The first type deals with the convergence of numerical solutions as the time becomes large. The second type shows that as the time and space increments $\Delta t$ and $\Delta x$ are allowed to approach zero, the numerical solutions become identical with the corresponding analytic solutions.

From an inspection of (2.1) and (2.2), it is seen that in applying numerical solutions to any particular example, there are apparently two arbitrary quantities, the space increment $\Delta x$, and the modulus $a$, which in turn determines the time increment $\Delta t$. However, it is found from experience that if the value of $a$ is taken too small, the calculated numerical answers oscillate and ultimately diverge as the time becomes large. The first type of convergence is concerned with developing criteria which impose a lower limit on allowable values of $a$ which will then insure numerical convergence. Each example considered has such a criterion developed, since such criteria usually depend on the particular boundary conditions. Another related problem is that of determining the steady-state distribution given numerically. It is shown that numerical solutions converge to the same steady-state values as those determined analytically for the boundary conditions under consideration.

Both of the problems discussed above pertain to actual numerical solutions where the space and time increments are finite, non-zero quantities. The second type of convergence is treated in $\$ 11$, apart from the main body of the paper, since it does not deal with numerical solutions as applied, but rather to the limiting case where $\Delta x$ and $\Delta t$ approach zero. Under these conditions, the numerical solutions become identical with the analytic solutions for all values of time and position throughout the slab.

4. Particular solutions and contour integration. By substituting $T_{x, t}=F(t) \sin z x$ into (2.1), $F(t)$ is found to satisfy the subsidiary difference equation $F(t)$ $=[(a+2 \cos z) /(a+2)] F(t-1)$ and therefore $F(t)=[(a+2 \cos z) /(a+2)] t$, from which we find as a particular solution to (2.1),

$$
T_{x, t}=\left(\frac{a+2 \cos z}{a+2}\right)^{t} \sin z x .
$$


A similar analysis shows that $\sin z x$ may be replaced by $\cos z x$ or $e^{i z x}$.

Using (4.1) and extending it to the two or three-dimensional form, we can write down immediately solutions in Fourier series and integrals for rectangular objects, with arbitrary initial temperature distributions. However, such solutions are of little use as they converge too slowly. Instead of following the standard Fourier development, the author has found it expedient to consider only cases where the initial temperature over the slab is zero (which by a change in temperature origin includes any constant temperature distribution). This restriction, for the one-dimensional slab, allows the use of a method of contour integration which may be summarized as follows.

a) Having found that

$$
T_{x, t}=\left(\frac{a+2 \cos z}{a+z}\right)^{t}\left[\frac{A(z)}{z} \cos z x+\frac{B(z)}{z} \sin z x\right]
$$

is a particular solution as long as $A(z)$ and $B(z)$ are independent of $x$ and $t$, we formally integrate this solution with respect to $z$ over the prescribed path (Fig. 2) in the complex plane. The solution is (4.2) below, where the functions $A(z)$ and $B(z)$ are determined so that (4.2) satisfies the boundary conditions of the problem.

$$
T_{x, t}=\frac{1}{\pi i} \int_{P}\left(\frac{a+2 \cos z}{a+2}\right)^{t}(A(z) \cos z x+B(z) \sin z x) \frac{d z}{z} .
$$

The path $P$ is chosen parallel to the real axis, extending from $+\infty$ to $-\infty$, and is

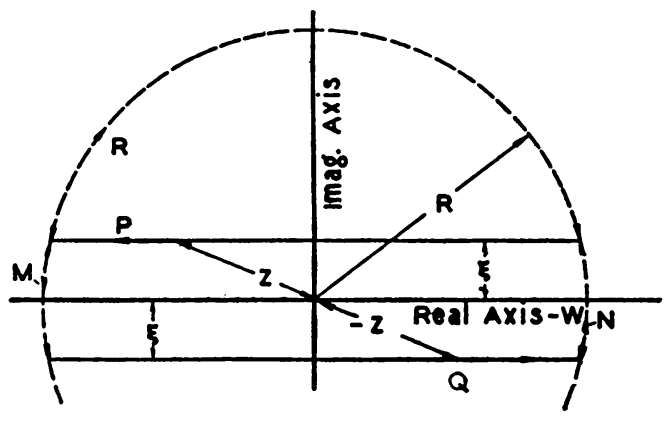

FIG. 2. located a finite distance $m$ above the real axis, $m$ being determined so that all poles of the integrand lie below $P$.

b) The integrand of (4.2) is shown to vanish over the arc, path $R$ of Fig. 2, except possibly at the slab boundary points $x=0$ or $x=l$, when $t$ is given the value zero. Then, as there are no poles enclosed by paths $P$ and $R$, it follows from Cauchy's theorem that at time zero $T_{x, 0}=0$, except possibly at the slab boundaries. It follows that (4.2) is the solution to the problem, for it satisfies the difference equation, the initial condition $T_{x, 0}=0$, and the boundary conditions.

c) The remaining step is the evaluation of the contour integral. This is accomplished in one of two ways. In either case, the integrand of (4.2) is shown to vanish over the paths $M$ and $N$ (Fig. 2) or over half these paths.

1) For semi-infinite slab problems, the integral over $P$ is evaluated in terms of an integral along the real axis and the residues of any poles lying between path $P$ and the real axis.

2) For finite slab problems, the functions $A(z)$ and $B(z)$ are generally such that the integrand is even-valued, and therefore the solution (4.2) may equally well be integrated over the path $Q$ (Fig. 2) which is opposite path $P$. Thus, integration around the loop consisting of paths $P$ and $Q$, and the paths $M$ and $N$ shows that the required 
integral is equal to half the sum of the residues at the poles enclosed by paths $P, Q$, $M$ and $N$, since the paths $M$ and $N$ contribute nothing to the integral over the loop.

All analysis of a purely rigorous nature has been omitted from the paper, but all doubtful cases have been tested for proper convergence and the vanishing of the integrals over the paths outlined above.

5. Semi-infinite slab. Boundary $x=0$ held at constant temperature $u_{0}$, initial temperature zero. Let us consider the following equation

$$
T_{x, t}=\frac{u_{0}}{\pi i} \int_{P}\left(\frac{a+2 \cos z}{a+2}\right)^{t} e^{i x x} \frac{d z}{z} .
$$

To prove that this expression is the solution of the problem it is necessary to show that $T_{x, 0}=0$ and that $T_{0, t}=u_{0}$.

To show that $T_{x, 0}=0$, we set $t=0$ and make the substitution $z=R e^{i \phi}$. We then integrate $\left(u_{0} / \pi\right) \exp [i x R \exp (i \phi)] d \phi$ over the path $R, \phi$ varying from $\pi$ to 0 . It follows that as $R \rightarrow \infty$ this integral vanishes, except at $x=0$. Therefore, since there are no poles enclosed by paths $P$ and $R$, it follows from Cauchy's theorem that $T_{x, 0}=0$.

To show that the integrand vanishes over the paths $M$ and $N$, let us substitute $z= \pm R+i y$ where $y$ varies from $-m$ to $+m$, and let $R \rightarrow \infty$; then it follows that these integrals vanish. $T_{0, t}$, the temperature at $x=0$, can equally well be integrated over path $Q$, since the resulting integrand is even-valued. Therefore, by Cauchy's theorem, since there are no contributions from the paths $M$ and $N, T_{0, t}=u_{0}$. It follows that (5.1) is the solution to the problem, for it satisfies the initial and boundary conditions.

To evaluate (5.1) it is convenient to integrate around the loop consisting of $P$, the half-path $M$, the real axis indented at the origin by a small semi-circle of radius $\epsilon$, and the half-path $N$. In the work that follows, the real axis will be denoted by $w$ to avoid confusion with the slab position variable.

Since the integrals over $M$ and $N$ vanish, and there are no poles enclosed in the loop,

$$
\begin{aligned}
T_{x, t} & =\frac{u_{0}}{\pi i} \int_{+\infty}^{+e}\left(\frac{a+2 \cos w}{a+2}\right)^{t} e^{-i x w} \frac{d w}{w} \\
& +\frac{u_{0}}{\pi} \int_{\pi}^{0}\left(\frac{a+2 \cos \epsilon e^{i \phi}}{a+2}\right)^{t} \exp [i x \epsilon \exp (i \phi)] d \phi \\
& +\frac{u_{0}}{\pi i} \int_{+}^{+\infty}\left(\frac{a+2 \cos w}{a+2}\right)^{t} e^{+i x w} \frac{d w}{w}=0 .
\end{aligned}
$$

Regrouping terms and letting $\epsilon \rightarrow 0$, we have as the solution

$$
T_{x, t}=u_{0}\left[1-\frac{2}{\pi} \int_{0}^{\infty}\left(\frac{a+2 \cos w}{a+2}\right)^{t} \sin w x \frac{d w}{w}\right] .
$$

To express this solution in terms of the polynomials, we expand $(a+2 \cos w)^{t}$ according to (12.7) and substitute into (5.2),

$$
T_{x, t}=u_{0}\left[1-\frac{2}{\pi(a+2)^{t}} \int_{0}^{\infty}\left[P_{t}(t)+2 P_{t-1}(t) \cos w+\cdots\right.\right.
$$

$$
\left.\left.+2 P_{0}(t) \cos t w\right] \sin w x \frac{d w}{w}\right] \text {. }
$$


Combining the sine and cosine terms and making use of the identity (12.6a), $P_{t-r}(t)=P_{t+r}(t)$, we have

$$
T_{x, t}=u_{0}\left[1-\frac{2}{\pi(a+2)^{t}} \int_{0}^{\infty} \sum_{r=-t}^{r+t} P_{r+t}(t) \sin w(x+r) \frac{d w}{w}\right]
$$

or

$$
T_{x, t}=u_{0}\left[1-\frac{1}{(a+2)^{t}} \sum_{r=-t}^{r+t}\{x+r\} P_{r+t}(t)\right]
$$

where the symbols $\{x+r\}=1,0$, or -1 depending on whether $x+r$ is greater than, equal to or less than zero. This notation arises from the fact that $(2 / \pi) \int_{0}^{\infty}(\sin w(x+r) / w) d w=\{x+r\}$ in accordance with the above convention.

From (5.2) it is easily shown that the solution converges as $t \rightarrow \infty$ when

$$
a \geqq 0 \text {. }
$$

To show that the numerical solution converges to the analytic steady-state solution for the same boundary conditions when $t \rightarrow \infty$, the following device is used: $T_{x, t}$ may be equated to the integrand over the real axis, and added to the sum of all residues of poles enclosed by the real axis and the path $P$. The real axis is to be indented with small semi-circles at all poles lying on it. In the steady-state value of the solution, the only contributions which remain as $t \rightarrow \infty$, are those which occur where $z=2 n \pi$. All other contributions, including those along the real axis, drop out due to the rapidity with which the factor $[(a+2 \cos z) /(a+2)]^{t} \rightarrow 0$ when $z \neq 2 n \pi$, as $t \rightarrow \infty$

In the problem under consideration, there are no poles, and if we take in to account the indentation at the origin, we find that the steady-state solution approaches $u_{0}$. This is also the value given by the analytic solution of the same problem.

Although simpler methods would have given the same result in this case, the method is very powerful, since it may be used on a solution with no further reduction from the contour integral form.

6. Semi-infinite slab, $T_{0, t}$ polynomial in time, initial temperature zero. Let us consider the following equation

$$
T_{x, t}=(-1)^{n} \frac{C(2 n) !}{2 \pi i} \int_{P}\left(\frac{a+2 \cos z}{a+2}\right)^{t} e^{i z x} z^{-(2 n+1)} d z .
$$

An analysis similar to that in $\$ 5$ shows that $T_{x, 0}=0$ and also that the integrand vanishes over the paths $M$ and $N$.

When $x=0$, the integrand is even-valued in $z$, and therefore has the same value over path $Q$ as over $P$. $T_{0, t}$ may then be equated to half the sum of the residues at the poles enclosed by paths $P$ and $Q$. From (12.8) this becomes

$$
T_{0, t}=\xi_{2 n}(t),
$$

where $\xi_{2 n}(t)$ is a polynomial in $t$ of the $n$th degree. From proper combinations of these polynomials, contour integrals are readily derived for problems in which the boundary temperatures are arbitrary polynomials in time.

For the particular case where the boundary temperature is linear in time and 
therefore given by $T_{0, t}=C \xi_{2}(t)=C t /(a+2)$, as follows from (12.10), the contour solution is given by,

$$
T_{x, t}=-\frac{C}{\pi i} \int_{P}\left(\frac{a+2 \cos z}{a+2}\right)^{t} e^{i z x} \frac{d z}{z^{3}} .
$$

We indent the origin, integrate as in $\$ 5$ to obtain

$$
\frac{2 C}{\pi} \int_{\epsilon}^{\infty}\left(\frac{a+2 \cos w}{a+2}\right)^{t} \sin w x \frac{d w}{w^{3}}-\frac{C}{\pi} \int_{0}^{\pi}\left(\frac{a+2 \cos \epsilon e^{i \pi}}{a+2}\right)^{t} \frac{\exp \left[i x \epsilon e^{i \phi}\right]}{\epsilon^{2} e^{2 i \phi}} d \phi
$$

The first integral, after the expansion of $(a+2 \cos w)^{t}$ in terms of the polynomials and regrouping as in (5.2), becomes

$$
I_{1}=\frac{2 C}{\pi(a+2)^{t}} \sum_{r=-t}^{r+t} \int_{0}^{\infty} \sin w(x+r) P_{r+t}(t) \frac{d w}{w^{3}} .
$$

By integrating by parts and keeping all terms which do not drop out as $\epsilon \rightarrow 0$, the contribution of this integral is found to be

$$
I_{1} \cong \frac{2 C x}{\pi \epsilon}-\frac{C}{\pi(a+2)^{t}} \sum_{r=-t}^{-+t}(x+r)^{2} P_{r+t}(t) \int_{\epsilon}^{\infty} \sin w(x+r) \frac{d w}{w} .
$$

The second integral is expanded in terms of $\epsilon$ and $\phi$, and all terms are retained which do not drop out at the integration limits or as $\epsilon \rightarrow 0$. We then have for this integral

$$
I_{2} \cong-\frac{2 C x}{\pi \epsilon}+\frac{C x^{2}}{2}+\frac{C t}{a+2}
$$

The sum of $I_{1}$ and $I_{2}$ with $\epsilon \rightarrow 0$ then gives for the solution

$$
T_{x, t}=C\left[\frac{t}{a+2}+\frac{x^{2}}{2}-\frac{1}{2(a+2)^{t}} \sum_{r=-t}^{r+t}(x+r)^{2} P_{r+t}(t)\{x+r\}\right],
$$

where the symbols $\{x+r\}$ have the same meaning as in (5.3).

An analysis similar to that of $\$ 5$ shows that if $a \geqq 0$, the numerical solution approaches $C t /(a+2)$ asymptotically as $t \rightarrow \infty$. This result also follows from the analytic theory.

7. Finite slab, length $l$, boundaries $x=0$ and $x=l$ held at constant temperatures $u_{0}$ and $u_{\imath}$ respectively, initial temperature zero. Let us consider the equation

$$
T_{x, t}=\frac{1}{\pi i} \int_{P}\left(\frac{a+2 \cos z}{a+2}\right)^{t}[A(z) \cos z x+B(z) \sin z x] \frac{d z}{z},
$$

where the functions $A(z)$ and $B(z)$ must be determined to make (7.1) satisfy the boundary conditions $T_{0, t}=u_{0}$ and $T_{l, t}=u_{l}$. Referring to (5.1) and placing $x=0$, we see that

$$
\frac{u_{0}}{\pi i} \int_{P}\left(\frac{a+2 \cos z}{a+2}\right)^{t} \frac{d z}{z}=u_{0} \text {. }
$$

Therefore if $A(z)=u_{0}$ and $B(z)=\left(u_{l}-u_{0} \cos z l\right) / \sin z l,(7.1)$ reduces to $u_{0}$ at $x=0$ and to $u_{l}$ at $x=l$. The solution is therefore 


$$
T_{x, t}=\frac{1}{\pi i} \int_{P}\left(\frac{a+2 \cos z}{a+2}\right)^{t}\left(u_{0} \cos z x+\frac{u_{l}-u_{0} \cos z l}{\sin z l} \sin z x\right) \frac{d z}{z} .
$$

The integrand of (7.3) is an even-valued function of $z$ and therefore the solution may be equated to one-half the sum of the residues at poles enclosed by paths $P$ and $Q$ (there are no contributions from the paths $M$ and $N$ ). Poles occur at $z=n \pi / l$, $n$ any integer. After evaluating the residues and simplifying the result, we have

$$
\begin{aligned}
T_{x, t}=u_{0} & +\left(u_{l}-u_{0}\right) \frac{x}{l} \\
& +\frac{2}{\pi} \sum_{1,2}^{\infty}(-1)^{n}\left(u_{l}-u_{0} \cos n \pi\right) \frac{\sin (n \pi x / l)}{n}\left(\frac{a+2 \cos (n \pi / l)}{a+2}\right)^{t} .
\end{aligned}
$$

To express this solution in terms of the polynomials, it is necessary to expand $(a+2 \cos (n \pi) / l)^{t}$ as in (12.7),

$$
\begin{aligned}
& T_{x, l}= u_{0}+\left(u_{l}-u_{0}\right) \frac{x}{l} \\
&+\frac{2}{\pi(a+2)^{t}} \sum_{1,2}^{\infty}(-1)^{n}\left(u_{l}-u_{0} \cos n \pi\right) \frac{\sin (n \pi x / l)}{n}\left[P_{t}(t)+2 P_{t-1}(t) \cos \frac{n \pi}{l}\right. \\
&\left.+\cdots+2 P_{0}(t) \cos \frac{n \pi t}{l}\right] .
\end{aligned}
$$

Combining the trigonometric terms and making use of the identity $P_{t-r}(t)=P_{t+r}(t)$, we obtain the solution in the form

$$
\begin{aligned}
T_{x, t}= & u_{0}+\left(u_{l}-u_{0}\right) \frac{x}{l} \\
& +\frac{2}{\pi(a+2)^{t}} \sum_{r=-t}^{r+t} P_{t+r}(t) \sum_{1,2}^{\infty} \frac{(-1)^{n}}{n}\left(u_{l}-u_{0} \cos n \pi\right) \sin \frac{n \pi(x+r)}{l} .
\end{aligned}
$$

However from the initial condition $T_{x, 0}=0$, it follows that at $t=0$, the resulting sine expansion of (7.4) must be equal to $-u_{0}-\left(u_{l}-u_{0}\right)(x / l)$. Therefore the infinite series in the double summation of (7.5) must equal $-F(x+r)$, where $F(x)$ is the periodic sine expansion of $u_{0}+\left(u_{l}-u_{0}\right)(x / l)$. The solution then becomes

$$
T_{x, t}=u_{0}+\left(u_{l}-u_{0}\right) \frac{x}{l}-\frac{1}{(a+2)^{t}} \sum_{r=-t}^{-+t} P_{r+t}(t) F(x+r) .
$$

Figure 3 shows the graph of the periodic sine expansion of $F(x)=u_{0}+\left(u_{l}-u_{0}\right)(x / l)$

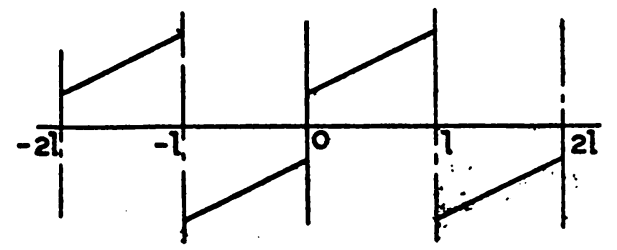

FIG. 3. which applies to (7.6). In applying (7.6) it is usually simpler to plot the function and then pick off the different values of $F(x)$ required in the summation. It will be noticed that when $x+r$ is a multiple of $l$, the value of $F(x+r)$ is zero.

For finite slab problems, it is usually possible to get a solution in the form of a finite Fourier series, in addition to the poly- 
nomial expansion. The derivation of such series is not difficult, but is too long to be given here in full. The finite series for $\$ 7$ is

$$
T_{x, l}=u_{0}+\left(u_{l}-u_{0}\right) \frac{x}{l}-\sum_{1}^{l-1} A_{n} \sin \frac{n \pi x}{l}\left(\frac{a+2 \cos (n \pi / l)}{a+2}\right)^{t},
$$

where

$$
A_{n}=\frac{\sin (n \pi / l)\left(u_{l}-u_{0} \cos n \pi\right)}{l(1-\cos (n \pi / l))} .
$$

The general method used for obtaining finite series, such as (7.7), consists of a replacement of the infinite Fourier series as in (7.4) by a finite number of terms of the same type, such that at $t=0$ the finite series reduces to the same function as given by the infinite series at $t=0$. Such expansions are possible since in numerical methods the initial temperature must be specified only at a finite number of points. The coefficients for the terms of such finite series are given by

$$
A=\frac{2}{l} \sum_{x=1}^{x=l} F(x) \sin \frac{n \pi x}{l}
$$

where $F(x)$ is the function at $t=0$ over the interval 0 to $l$. The $A_{n}$ of (7.8) were calculated as above with $F(x)=u_{0}+\left(u_{l}-u_{0}\right)(x / l)$.

From an inspection of (7.7) we see that as $t \rightarrow \infty$ the solution converges provided $|\{a+2 \cos (n \pi / l)\} /(a+2)| \leqq 1$. A simple analysis yields the following criterion for convergence

$$
\begin{aligned}
& \text { 1) } a+2 \geqq 2 \cos ^{2}(\pi / 2 l) \quad l \text { even, } \\
& \text { 2) } a+2 \geqq 2 \cos ^{2}(\pi / l) \quad l \text { odd. }
\end{aligned}
$$

Equation (7.7) also shows that the numerical solution approaches $u_{0}+\left(u_{l}-u_{0}\right)(x / l)$ as $t \rightarrow \infty$, which is the analytic steady-state solution for the same boundary conditions.

8. Finite slab, length $l$, insulated at $x=0$, held at constant temperature $u_{l}$ at $x=l$, initial temperature zero. The boundary conditions are

$$
T_{l, t}=u_{l}
$$

and from Eq. (2.7),

$$
T_{0, t}=\frac{2 T_{1, t-1}+a T_{0 t-1}}{a+2}
$$

Imposing these conditions on the general contour integral (4.2), we find that $A(z)=u_{l} / \cos z l$ and $B(z)=0$. The solution is therefore

$$
T_{x, t}=\frac{u_{l}}{\pi i} \int_{P}\left(\frac{a+2 \cos z}{a+2}\right)^{t} \frac{\cos z x}{\cos z l} \frac{d z}{z} .
$$

Poles occur at $z=0$ and $z=(2 n+1) \pi / 2 l$. Evaluating the integral as in $\S 7$ and regrouping terms, we obtain the solution

'W. E. Byerly, Fourier's series and spherical, cylindrical and ellipsoidal harmonics, Ginn Co., Boston, 1895. pp. 30-35. 


$$
T_{x, t}=u_{l}\left[1+\frac{4}{\pi} \sum_{1,3}^{\infty}(-1)^{(n+1) / 2}\left(\frac{a+2 \cos (n \pi / 2 l)}{a+2}\right)^{t} \frac{\cos (n \pi x / 2 l)}{n}\right] .
$$

An analysis similar to that of $\$ 7$ yields the polynomial expansion

$$
T_{x, t}=u_{\imath}\left[1-\frac{1}{(a+2)^{t}} \sum_{r=-t}^{r=+t} P_{r+t}(t) F(x+r)\right]
$$

where $F(x)$ is the cosine Fourier expansion of unity from 0 to $l$ and minus unity from $l$ to $2 l$, as is evident from (8.4) and the initial condition $T_{x, 0}=0$. It will be noted that the value of $F(x+r)$ is zero when $x+r$ is an odd multiple of $l$.

The finite Fourier series solution is found to be

$$
T_{x, t}=u_{l}\left[1+\sum_{1}^{2 l-1} A_{n} \sin \frac{n \pi(x-l)}{2 l}\left(\frac{a+2 \cos (n \pi / 2 l)}{a+2}\right)^{t}\right],
$$

where

$$
A_{n}=0 \quad n \text { even, } \quad A_{n}=\frac{\sin (n \pi / 2 l)}{l(1-\cos (n \pi / 2 l))} \quad n \text { odd. }
$$

From (8.6) it follows that the convergence criterion is:

$$
\begin{aligned}
& \text { 1) } a+2 \geqq 2 \cos ^{2}(\pi / 4 l) \quad l \text { even, } \\
& \text { 2) } a+2 \geqq 2 \cos ^{2}(\pi / 2 l) \quad l \text { odd. }
\end{aligned}
$$

Also from (8.6) it follows that as $t \rightarrow \infty$, the numerical steady-state solution becomes $u_{l}$ which is also the analytic steady-state solution for the same problem.

9. Finite slab, length $l$, constant energy input $q$ at $x=l$, temperature kept at zero at $x=0$, initial temperature zero. The boundary conditions are (from Eq. (2.5))

$$
T_{l, t}=\frac{2 T_{l-1, t-1}+a T_{l, t-1}+2 Q}{a+2}, \quad \text { (9.1) } \quad T_{0, t}=0
$$

where $Q=q \Delta x / k$. Imposing these conditions on (4.2) we find that $A(z)=0$ and $B(z)=Q /(\sin z \cos z l)$. The solution is therefore

$$
T_{x, t}=\frac{Q}{\pi i} \int_{P} \frac{\sin z x}{\sin z \cos z l}\left(\frac{a+2 \cos z}{a+2}\right)^{t} \frac{d z}{z} .
$$

Poles occur at $z=0$ and $z=(2 n+1) \pi / 2 l$ (the set $z=n \pi, n \neq 0$ does not constitute poles, as the terms $\sin z x$ in the numerator also vanish at these points since $x$ is restricted to integral values). After evaluating the residues, regrouping terms and simplifying, we see that the solution becomes

$$
T_{x, t}=Q\left[x+\frac{4}{\pi} \sum_{1,3}^{\infty}(-1)^{(n+1) / 2} \frac{\sin (n \pi x / 2 l)}{n \sin (n \pi / 2 l)}\left(\frac{a+2 \cos (n \pi / 2 l)}{a+2}\right)^{t}\right] .
$$

The polynomial expansion is found to be,

$$
T_{x, t}=Q\left[1-\frac{1}{(a+2)^{t}} \sum_{r=-t}^{r+t} P_{r+t}(t) F(x+r)\right]
$$


where $F(x)$ is the sine expansion of $x$ from 0 to $l$ and of $2 l-x$ from $l$ to $2 l$, as follows from (9.4) and the initial condition $T_{x, 0}=0$.

The finite Fourier series is

$$
T_{x, t}=Q\left[x-\sum_{1}^{2 l-1} A_{n} \sin \frac{n \pi x}{2 l}\left(\frac{a+2 \cos (n \pi / 2 l)}{a+2}\right)^{\natural}\right],
$$

where

$$
A_{n}=0 \quad n \text { even, } \quad A_{n}=\frac{(-1)^{(n-1) / 2}}{l(1-\cos (n \pi / 2 l))} \quad n \text { odd } .
$$

From (9.6) the convergence criterion is found to be

$$
\begin{aligned}
& \text { 1) } a+2 \geqq 2 \cos ^{2} \frac{\pi}{4 l} \quad l \text { even, } \\
& \text { 2) } a+2 \geqq 2 \cos ^{2} \frac{\pi}{2 l} \quad l \text { odd. }
\end{aligned}
$$

From (9.6) it also follows that as $t \rightarrow \infty$ the numerical solution approaches $Q x$, which is equivalent to the analytic steady-state solution for the same boundary conditions.

10. Convection at a boundary. When the condition (2.3) is imposed on the general contour integral (4.2), $A(z)$ and $B(z)$ are generally such that the evaluation of the resulting integrals is difficult, due either to the uncertain nature of the poles, or to the evaluation of a complicated infinite integral.

For the semi-infinite slab with convection into temperature $T_{a}$, transfer coefficient $h$, the boundary condition is given by (2.3)

$$
T_{0, t}=\frac{2 T_{1, t-1}+(a-2 N) T_{0, t-1}+2 N T_{0}}{a+2}, \quad N=h \Delta x / k .
$$

We assume a contour integral solution of the form,

$$
T_{x, t}=\frac{1}{\pi i} \int_{P} A(z)\left(\frac{a+2 \cos z}{a+2}\right)^{t} e^{i z x} \frac{d z}{z} .
$$

Imposing the condition (10.1) on (10.2) we see that $A(z)=N T_{a} /(N-i \sin z)$, and the solution may therefore be written,

$$
T_{x, t}=\frac{N T_{a}}{\pi i} \int_{P}\left(\frac{a+2 \cos z}{a+2}\right)^{t} \frac{e^{i z x}}{N-i \sin z} \frac{d z}{z} .
$$

The integral (10.3) is to be evaluated in terms of an integral along the real axis indented at the origin, plus the sum of the residues at the poles enclosed by the path $P$ and the real axis. Aside from the root $z=0$, the denominator includes roots from the term $N-i \sin z$, which are found to be $z=-i \log \left(\sqrt{N^{2}+1}+N\right)$ and the infinite set $z=-i \log \left(\sqrt{N^{2}+1}-N\right)+(2 n+1) \pi, n$ any integer. However, in the loop of integration considered, only the residues at the poles $z=-i \log \left(\sqrt{N^{2}+1}-N\right)+(2 n+1) \pi$ are evaluated, since the other poles lie outside the loop. ( $N$ is taken greater than zero, otherwise the boundary would be insulated and no heat would flow, giving the trivial solution $T_{x, t} \equiv 0$.) 
Following the analysis of (5.1), integrating along the indented real axis, and then letting $\epsilon \rightarrow 0$, we obtain the solution in the form

$$
\begin{aligned}
T_{x, t}= & T_{a}\left[1-\frac{2 N}{\pi} \int_{0}^{\infty}\left(\frac{a+2 \cos w}{a+2}\right)^{t}\left(\frac{N \sin w x+\sin w \cos w x}{N^{2}+\sin ^{2} w}\right) \frac{d w}{w}\right. \\
& \left.+2 \pi i \sum \text { residues }\right] .
\end{aligned}
$$

By a somewhat tedious but straightforward analysis, the residue term may be evaluated and simplified to yield,

$$
\begin{aligned}
2 \pi i \sum \text { Res. }=\frac{4 N T_{a} \log \left(\sqrt{N^{2}+1}-N\right)}{\sqrt{N^{2}+1}} & \left(\frac{a-2 \sqrt{N^{2}+1}}{a+2}\right)^{t}\left(\sqrt{N^{2}+1}-N\right)^{x} \\
& \cdot \sum_{1,3}^{\infty} \frac{\cos n \pi x}{n^{2} \pi^{2}+\log ^{2}\left(\sqrt{N^{2}+1}-N\right)} .
\end{aligned}
$$

By choosing a combination of known Fourier expansions for the hyperbolic sine and cosine, with further reduction, and recalling that in numerical analysis $x$ is always an integer, we can write (10.5) in the form

$$
2 \pi i \sum \text { Res. }=-T_{a}\left(\frac{a-2 \sqrt{N^{2}+1}}{a+2}\right)^{\prime}\left(N-\sqrt{\left.N^{2}+1\right)} \times\left(\frac{\sqrt{N^{2}+1}-1}{\sqrt{N^{2}+1}}\right) .\right.
$$

The final solution therefore becomes

$$
\begin{aligned}
T_{x, l}=T_{a} & {\left[1-\frac{2 N}{\pi} \int_{0}^{\infty}\left(\frac{a+2 \cos w}{a+2}\right)^{t}\left(\frac{N \sin w x+\sin w \cos w x}{N^{2}+\sin ^{2} w}\right) \frac{d w}{w}\right.} \\
& \left.-\left(\frac{\sqrt{N^{2}+1}-1}{\sqrt{N^{2}+1}}\right)\left(\frac{a-2 \sqrt{N^{2}+1}}{a+2}\right)^{t}\left(N-\sqrt{N^{2}+1}\right)^{x}\right] .
\end{aligned}
$$

Without evaluating the infinite integral (which is convergent when $t \rightarrow \infty$ provided $a \geqq 0)$ we see by inspection of the factor $\left[\left(a-2 \sqrt{N^{2}+1}\right) /(a+2)\right]^{t}$ that the criterion for convergence as $t \rightarrow \infty$ is,

$$
a \geqq \sqrt{N^{2}+1}-1
$$

It will be noted that Schmidt's equation, where $a=0$, will not yield convergent answers if the boundary expression (2.3) is used, since by (10.7) $a$ cannot be zero. It also follows from (10.6) that the numerical steady-state solution becomes $T_{a}$, which is also the solution predicted analytically for the same problem.

A polynomial expansion may be obtained from (10.6) by using (12.7) to expand the term $(a+2 \cos w)^{t}$. However, the analysis required to evaluate the resulting definite integrals is so involved that it is not worthwhile to include the expansion here.

Contour integral solutions are readily set up for finite slab problems involving convection at either or both boundaries, but it is generally difficult to evaluate these integrals. However, without evaluating the integrals, but using the method outlined 
in (5.3), we can show that if the numerical work converges at all, it converges to the correct steady-state distribution as $t \rightarrow \infty$.

11. Convergenre to analytic solution. The theory developed so far has been concerned only with numerical solutions as actually applied. The convergence problems already discussed have shown what values of $a$ are necessary to insure non-divergent numerical answers, and also that as the time increases, numerical solutions approach the same steady-state distribution as that given analytically for the same problem. These results hold when the space and time increments $\Delta x$ and $\Delta t$ are finite, non-zero quantities.

It is also possible to show that as the arbitrary increments $\Delta x$ and $\Delta t$ become very small, the numerical solutions approach the true analytic solution for all values of $x$ and $t$, and attain this solution in the limit. The formal procedure used to demonstrate this limiting convergence consists of a demonstration that the contour integrals derived for the numerical solutions transform into already known contour integral solutions for the corresponding analytic treatment, as $\Delta x$ and $\Delta t$ approach zero. Formal proofs of this convergence will be given for three of the numerical examples already discussed. The proofs for other examples are very similar to the ones given here.

The three examples considered with their analytic contour integral solutions are: 6

1) Semi-infinite slab, end $x^{\prime}=0 \mathrm{kept}$ at temperature $u_{0}$, initial temperature zero,

$$
T\left(x^{\prime}, t^{\prime}\right)=\frac{u_{0}}{\pi i} \int_{P} e^{i z x^{\prime}} e^{-\alpha z^{2} t^{\prime}} \frac{d z}{z} .
$$

2) Semi-infinite slab, convection at $x^{\prime}=0$ into a medium of constant temperature $T_{a}$, initial temperature zero,

$$
T\left(x^{\prime}, t^{\prime}\right)=\frac{h T_{a}}{k \pi i} \int_{P} \frac{e^{i z x^{\prime}} e^{-\alpha z^{2} t^{\prime}}}{(h / k)-i z} \frac{d z^{\prime}}{z^{\prime}} .
$$

3) Finite slab, length $l^{\prime}$, end $x^{\prime}=0$ held at zero temperature, constant energy input $q$ at $x^{\prime}=l^{\prime}$, initial temperature zero,

$$
T\left(x^{\prime}, t^{\prime}\right)=\frac{q}{k \pi i} \int_{P} \frac{\sin z x^{\prime}}{\cos z l^{\prime}} e^{-\alpha z z^{\prime}} \frac{d z}{z^{2}} .
$$

Equation (11.3) is not given in Ref. 6, but we can easily derive it by imposing the boundary condition $\partial T / \partial x-q / k=0$ on the general contour integral considered there.

The notation used in the above equations differs from that used in the present paper. In order to express these equations in our notation, it is necessary that the complex variable $\alpha$ be replaced by $z$, thermal diffusivity $K$ by $\alpha$, and convection coefficient $h$, by $h / k$.

$T\left(x^{\prime}, t^{\prime}\right)$ has been used to denote the analytic solution at the point $x^{\prime}$ and the time $t^{\prime}$, as distinguished from $T_{x, t}$, the numerical solution at point $x$ (in units of $\Delta x$ ) and time $t$ (in units of $\Delta t$ ). The path $P$ is the same for both numerical and analytic solutions, and is the limiting path allowable for the analytic integrals.

In the application of numerical solutions, position and time variables as well as slab lengths are expressed in terms of the arbitrary time and space increments $\Delta t$ and $\Delta x$. In order to discuss the convergence to analytic solutions, it is necessary to express these quantities in terms of the absolute units used analytically. If $M$ arbi-

${ }^{6}$ H. S. Carslaw, The conduction of heat, Macmillan Co., New York, ed. 2, 1921, pp. 97-99. 
trary space increments equal one absolute unit, then the absolute space position $x^{\prime}$ is given by,

$$
x^{\prime}=k / M \quad \text { or } \quad x=M x^{\prime} .
$$

From the relationship $\Delta t=(\Delta x)^{2} /(a+2) \alpha$ of $(2.2)$, it follows that the time value in absolute units is

$$
t^{\prime}=t / M^{2}(a+2) \alpha \text { or } t=M^{2}(a+2) \alpha t^{\prime} .
$$

To show that the numerical solution approaches the analytic solution for Case (1), when $\Delta x$ and $\Delta t$ approach zero, we substitute (11.4) and (11.5) into the numerical solution (5.1), obtaining

$$
T_{x, t}=\frac{u_{0}}{\pi i} \int_{P}\left(\frac{a+2 \cos z}{a+2}\right)^{M 2(a+2) \alpha t^{\prime}} e^{i z M x^{\prime}} \frac{d z}{z} .
$$

By replacing the variable $z$ by $z^{\prime} / M$, we can write (11.6) in the form

$$
T_{x, t}=\frac{u_{0}}{\pi i} \int_{P}\left(\frac{a+2 \cos \left(z^{\prime} / M\right)}{a+2}\right)^{M z(a+2) \alpha t} e^{i z^{\prime} x^{\prime}} \frac{d z^{\prime}}{z^{\prime}} .
$$

The factor $\left[\left(a+2 \cos z^{\prime} / M\right) /(a+2)\right]^{M^{2}(a+2) \alpha t^{\prime}}$ may be approximated by $\left[1-\left(z^{\prime}\right)^{2}\right.$ $\left./(a+2) M^{2}\right]^{M^{2}(a+2) \alpha t^{\prime}}$ when $M$ is large, and in the limit becomes exp $\left[-\left(z^{\prime}\right)^{2} \alpha t^{\prime}\right]$. The limit of the numerical expression (11.7) as $\Delta x$ and $\Delta t$ approach zero (or as $M \rightarrow \infty$ ) therefore becomes,

$$
\underset{\lim \Delta x \rightarrow 0}{T_{x, t}}=\frac{u_{0}}{\pi i} \int_{P} e^{i z^{\prime} x^{\prime}} e^{-\alpha\left(z^{\prime}\right) z z^{\prime}} \frac{d z^{\prime}}{z^{\prime}}
$$

which is identical with the analytic solution (11.1).

With convection at a boundary or constant energy input, the values $N=h \Delta x / k$ of (2.4) and $Q=q \Delta x / k$ of (2.6) become,

$$
N=\frac{h}{k M}
$$

$$
Q=\frac{q}{k M}
$$

In Case (2) after substituting (11.4), (11.5) and (11.9) into the numerical solution (10.3), and then replacing the variable $z$ by $z^{\prime} / M$, we obtain

$$
T_{x, t}=\frac{h T_{a}}{k \pi i M} \int_{P}\left(\frac{a+2 \cos \left(z^{\prime} / M\right)}{a+2}\right)^{M z(a+2) \alpha t^{\prime}} \cdot \frac{e^{i z^{\prime} x^{\prime}}}{(h / k M)-i \sin \left(z^{\prime} / M\right)} \frac{d z^{\prime}}{z^{\prime}} .
$$

As before, the term $\left[\left(a+2 \cos z^{\prime} / M\right) /(a+2)\right]^{M^{2}(a+2) \alpha t^{\prime}}$ becomes $\exp \left[-\left(z^{\prime}\right)^{2} \alpha t^{\prime}\right]$ in the limit. The term $i \sin \left(z^{\prime} / M\right)$ may be approximated by $i z^{\prime} / M$ when $M$ is large, We make these changes, cancel the $M$ outside the integral with those in the term $(h / k M)-\left(L z^{\prime} / M\right)$, and let $M$ approach infinity; then (11.11) becomes

$$
\underset{\lim \Delta x \rightarrow 0}{T_{x, t}}=\frac{h T_{a}}{k \pi i} \int_{P} \frac{e^{i z^{\prime} x^{\prime}} e^{-\left(z^{\prime}\right) z^{\prime} \alpha t^{\prime}}}{(h / k)-i z^{\prime}} \frac{d z^{\prime}}{z^{\prime}}
$$

which is the analytic expression (11.2).

In Case (3), after substituting in (9.3) from (11.4), (11.5), (11.10), introducing the absolute length $l^{\prime}$ given by $l=M l^{\prime}$, and changing the variable $z$ to $z^{\prime} / M$, we obtain

$$
T_{x, t}=\frac{q}{k \pi i M} \int_{P} \frac{\sin z^{\prime} x^{\prime}}{\sin \left(z^{\prime} / M\right) \cos z^{\prime} l^{\prime}}\left(\frac{a+2 \cos \left(z^{\prime} / M\right)}{a+2}\right)^{M 2(a+2) \alpha t^{\prime}} \frac{d z^{\prime}}{z^{\prime}} .
$$


In the limit, the term $\sin \left(z^{\prime} / M\right)$ may be replaced by $z^{\prime} / M$ and as in (11.11), the solution may be written in the form

$$
\underset{\lim \Delta x \rightarrow 0}{T_{x, t}}=\frac{q}{k \pi i} \int_{P} \frac{\sin z^{\prime} x^{\prime}}{\cos z^{\prime} l^{\prime}} e^{-\left(z^{\prime}\right) 2 \alpha t^{\prime}} \frac{d z^{\prime}}{\left(z^{\prime}\right)^{2}},
$$

which is identical with the analytic solution (11.3).

It will be noted that the restrictions on $a$ as given by (9.8) become $a \geqq 0$ when $l$ (the number of units of $\Delta x$ in the slab) becomes large. Hence the proof for this convergence to the analytic solution holds only when $a \geqq 0$. An analysis of the other examples treated in this paper shows that for this limiting convergence, all criteria reduce to $a \geqq 0$.

12. Appendix. Properties of the polynomials $P_{r}(t)$. The polynomials $P_{r}(t)$ are defined as the coefficients of $z^{r}$ in the expansion of the trinomial $\left(1+a z+z^{2}\right)^{t}$, and are therefore functions of $r, t$ and the modulus $a$. Two identities follow readily, the first by definition, the second by setting $z=1$ in (12.1),

$$
\begin{aligned}
& \sum_{r=-t}^{r=+t} P_{r+t}(t) z^{r+t}=\left(1+a z+z^{2}\right)^{t} \\
& \sum_{r=-t}^{r+t} P_{r+t}(t)=(a+2)^{t} .
\end{aligned}
$$

By expanding the trinomial in the form $\left[(1+a z)+z^{2}\right]^{t}$ and collecting coefficients, we obtain an explicit formula for $P_{r}(t)$,

$$
P_{r}(t)=\left(\begin{array}{l}
t \\
r
\end{array}\right)\left(\begin{array}{l}
t \\
0
\end{array}\right) a^{r}+\left(\begin{array}{c}
t-1 \\
r-2
\end{array}\right)\left(\begin{array}{l}
t \\
1
\end{array}\right) a^{r-2}+\left(\begin{array}{c}
t-2 \\
r-4
\end{array}\right)\left(\begin{array}{l}
t \\
2
\end{array}\right) a^{r-4}+\cdots
$$

The polynomials may be expressed as definite integrals in the following way. By definition and Cauchy's theorem

$$
P_{r}(t)=\frac{1}{2 \pi i} \int_{C}\left(1+a z+z^{2}\right)^{t} \frac{d z}{z^{r+1}}
$$

where $C$ is a simple closed contour about the origin. By the choice of $C$ as a circle of unit radius, center at the origin, it follows that $z=e^{i \phi}$ and

$$
P_{r}(t)=\frac{1}{2 \pi} \int_{0}^{2 \pi}(a+2 \cos \phi)^{t} \cos (t-r) \phi d \phi .
$$

Writing the equality $\left(1+a z+z^{2}\right)^{t}=\left(1+a z+z^{2}\right)^{t-1}\left(1+a z+z^{2}\right)$ and collecting coefficients of $z^{r}$ on both sides of the equation, we have the following recursion formula, which may be used for rapid calculation of the polynomials,

$$
P_{r}(t)=P_{r}(t-1)+a P_{r-1}(t-1)+P_{r-2}(t-1) .
$$

From (12.3), (12.4) and (12.5), it follows that

$$
\begin{gathered}
P_{t-r}(t)=P_{t+r}(t), \\
P_{0}(0)=1, \quad P_{0}(t)=1, \quad P_{r}(0)=0, \quad r \neq 0 .
\end{gathered}
$$

As an example, the polynomials up to $t=3$ have been worked out for modulus $a=3$ using (12.5) and (12.6), and are shown in Table 1. Thus, $P_{4}(3)=30$ and $P_{2}(2)$ $=11$. 
TABLE 1.

\begin{tabular}{l|l|l|r|r|r|r|r}
\hline \hline$t r r$ & 0 & 1 & 2 & 3 & 4 & 5 & 6 \\
\hline 0 & 1 & & & & & & \\
1 & 1 & 3 & 1 & & & & \\
2 & 1 & 6 & 11 & 6 & 1 & & \\
3 & 1 & 9 & 30 & 45 & 30 & 9 & 1 \\
\hline
\end{tabular}

To construct a polynomial array, we start with $P_{0}(0)=1$. The polynomials following are calculated successively by use of the recursion formula (12.5). As a specific example, from the formula $P_{3}(3)=P_{3}(2)+a P_{2}(2)+P_{1}(2)$, we have on substituting the values presumably already calculated for $t=2, P_{3}(3)=6+3 \times 11+6=45$.

An important identity may be established as follows: we let $(a+2 \cos \theta)^{t}$ $=\sum_{n=0}^{t} A_{n} \cos n \theta$. From (12.4),

$$
P_{r}(t)=\int_{0}^{2 \pi} \sum_{n=0}^{t} A_{n} \cos n \phi \cos (t-r) \phi d \phi,
$$

from which it follows that $A_{t-r}=2 P_{r}(t), r \neq t$, and $A_{0}=P_{t}(t)$. Therefore

$$
\begin{aligned}
(a+2 \cos \theta)^{t}= & P_{t}(t)+2 P_{t-1}(t) \cos \theta+\cdots+2 P_{t-r}(t) \cos r \theta+\cdots \\
& +2 P_{0}(t) \cos t \theta .
\end{aligned}
$$

The polynomials $\xi_{2 n}(t)$ are defined by,

$$
\xi_{2 n}(t)=\frac{(-1)^{n}}{2} \frac{(2 n) !}{2 \pi i} \int_{C}\left(\frac{a+2 \cos z}{a+2}\right)^{t} \frac{d z}{z^{2 n+1}},
$$

where $C$ is a simple closed contour about the origin. By Cauchy's theorem, and evaluation of the residue at the pole $z=0,(12.8)$ becomes,

$$
\xi_{2 n}(t)=\frac{(-1)^{n}}{2}\left[\frac{d^{n}}{d \theta^{n}}\left(\frac{a+2 \cos \theta}{a+2}\right)^{n}\right]_{0-0} .
$$

The first three polynomials, evaluated from (12.9) are,

$$
\left.\begin{array}{l}
\xi_{2}(t)=\left(\begin{array}{l}
t \\
1
\end{array}\right)(a+2)^{-1}, \\
\xi_{4}(t)=\left(\frac{t}{1}\right)(a+2)^{-1}+12\left(\begin{array}{l}
t \\
2
\end{array}\right)(a+2)^{-2}, \\
\xi_{6}(t)=\left(\begin{array}{l}
t \\
1
\end{array}\right)(a+2)^{-1}+60\left(\begin{array}{l}
t \\
2
\end{array}\right)(a+2)^{-2}+360\left(\begin{array}{l}
t \\
1
\end{array}\right)(a+2)^{-3} .
\end{array}\right\}
$$

Acknowledgments. The author is indebted to Cmdr. G. M. Dusinberre, of the Marine Engineering Department, U.S. Naval Academy, for suggesting the paper and for many valuable ideas concerning boundary conditions, and to Lt. H. G. Elrod of the same department for reading the paper. The author is also grateful to Prof. H. W. Emmons of the Harvard University Graduate School of Engineering for reading the paper carefully, and for suggesting the convergence problem of Section 11. 